\title{
Influence of local strain heterogeneity on high piezoelectricity in $\mathrm{Ba}\left(\mathrm{Zr}_{0.2} \mathrm{Ti}_{0.8}\right) \mathrm{O}_{3}-50\left(\mathrm{Ba}_{0.7} \mathrm{Ca}_{0.3}\right) \mathrm{TiO}_{3}$ ceramics
}

\author{
Le Zhang, ${ }^{1,2,{ }^{*}}$ Xiaobing Ren, ${ }^{1}$ and Michael Carpenter ${ }^{2}$ \\ 1.Multidisciplinary Material Research Center, State Key Laboratory for Mechanical Behavior \\ of Material, Frontier Institute of Science and Technology, Xi'an Jiaotong University, Xi'an, \\ 710049, China \\ 2.Department of Earth Sciences, University of Cambridge, Downing Street, Cambridge, CB2 \\ 3EQ, UK \\ *corresponding author:zhangleking005@gmail.com;
}

\begin{abstract}
Dielectric and mechanical spectroscopies have been used to investigate ferroelectric transitions and twin wall dynamics in the lead-free ceramic $\mathrm{Ba}\left(\mathrm{Zr}_{0.2} \mathrm{Ti}_{0.8}\right) \mathrm{O}_{3^{-}}$ $50\left(\mathrm{Ba}_{0.7} \mathrm{Ca}_{0.3}\right) \mathrm{TiO}_{3}$ (BZT-50BCT), which is known to have a high piezoelectric coefficient $\left(d_{33}>545 \mathrm{pC} / \mathrm{N}\right)$. Results from dynamical mechanical analysis in the frequency range 0.2-20 $\mathrm{Hz}$ and resonant ultrasound spectroscopy in the frequency range $\sim 0.1-1.2 \mathrm{MHz}$ confirm the existence of three phase transitions with falling temperature, at $~ 360 \mathrm{~K}$ (cubic-tetragonal), 304 K (tetragonal-orthorhombic) and $273 \mathrm{~K}$ (orthorhombic-rhombohedral). In comparison with $\mathrm{BaTiO}_{3}$, however, the transitions are marked by rounded rather than sharp minima in the shear modulus. The pattern of acoustic loss is also quite different from that shown by $\mathrm{BaTiO}_{3}$ in having a broad interval of high loss at low temperatures, consistent with a spectrum of relaxation times for interactions of ferroelastic twin walls. Differences in the dielectric properties also suggest more relaxor-like characteristics for BZT-50BCT. It is proposed that the overall pattern of behavior is significantly influenced by strain heterogeneity at a local length scale in the perovskite structure due to the substitution of cations with different ionic
\end{abstract}


radii. The existence of this strain heterogeneity and its influence on the elastic behavior near the transition points could be contributory factors to the development of adaptive nanoscale microstructures and enhanced piezoelectric properties.

\section{Introduction}

Research on lead-free piezoelectric materials has attracted much attention in academia and industry due to restrictions on $\mathrm{Pb}$ in devices such as sensors, actuators, and transducers.[1-3] However, the inferiority of their properties has proved to be a serious limitation. The discovery of large piezoelectric coefficients in (1- $x) \mathrm{Ba}\left(\mathrm{Zr}_{0.2} \mathrm{Ti}_{0.8}\right) \mathrm{O}_{3}-x\left(\mathrm{Ba}_{0.7} \mathrm{Ca}_{0.3}\right) \mathrm{TiO}_{3}$ ceramics (BZT- $x \mathrm{BCT}, x$ is the molar percentage of BCT) has overcome this limitation through the use of advantageous properties which develop in association with morphotropic phase boundaries (MPBs).[4-10] A piezoelectric coefficient of $\sim 600 \mathrm{pC} / \mathrm{N}$ at room temperature at BZT-50BCT exceeds that of many widely used lead-based piezoelectric materials, such as $\mathrm{Pb}\left(\mathrm{Zr}_{1-x} \mathrm{Ti}_{x}\right) \mathrm{O}_{3}-5 \mathrm{~A}$ and $\mathrm{Pb}\left(\mathrm{Zr}_{1-x} \mathrm{Ti}_{x}\right) \mathrm{O}_{3}-5 \mathrm{H}$.[4]

Recent dielectric and mechanical spectroscopy investigations have revealed that there are two phase transitions at this MPB, tetragonal (T, P4mm) - orthorhombic (O, Amm2) rhombohedral (R, $R 3 m$ ), rather than the previously proposed single T-R transition.[11-18] At least part of the high piezoelectric effect in BZT-50BCT might also be attributable to the mobility of twin walls in nanoscale adaptive microstructures associated with the phase boundaries.[19-21] It must be expected that extrinsic and intrinsic effects associated with the phase transitions, the adaptive microstructures and twin wall motion could be distinguished on the basis of measurements of both dielectric and elastic responses at different frequencies. The primary objective of the present study was therefore to compare the dielectric permittivity and dielectric loss, measured at a range of standard frequencies $(60 \mathrm{~Hz}-100 \mathrm{kHz})$ for a sample of BZT-50BCT, with elastic moduli and anelastic loss for the same ceramic sample measured at two different frequencies, in the vicinity of $10 \mathrm{~Hz}$ by Dynamical Mechanical Analysis (DMA) and in the vicinity of $1 \mathrm{MHz}$ by Resonant Ultrasound Spectroscopy (RUS). There are the expected differences in response from $90^{\circ}$ twin walls, which are both ferroelectric and ferroelastic, and $180^{\circ}$ walls which are only ferroelectric, but more insightful results have come from a comparison with similar data collected from $\mathrm{BaTiO}_{3}$ (BTO). An important 
feature of BZT-50BCT appears to be that it has properties which are influenced by the presence of local strain heterogeneities on a unit cell scale due to the substitution of cations with significantly different ionic radii on both the A and B sites.

\section{Sample preparation and experimental methods}

BZT-50BCT and BTO ceramic samples were fabricated by a conventional solid-phase reaction method, starting with $\mathrm{BaZrO}_{3}$ (99.8\%), $\mathrm{CaCO}_{3}$ (99.9\%), $\mathrm{BaCO}_{3}$ (99.95\%), and $\mathrm{TiO}_{2}$ (99.9\%). Calcining at $1623 \mathrm{~K}$ for 3 hours, followed by sintering of pressed pellets at 1723-1773 K for 3 hours in air, produced ceramic discs with diameter $\sim 10 \mathrm{~mm}$ and thickness $\sim 1.5 \mathrm{~mm}$. Opposing faces of a BZT-50BCT disc were coated with silver and the temperature dependence of the permittivity $\mathcal{E}_{\mathrm{r}}$ determined using a HIOKI 3532 LCR meter at testing frequencies of $0.06,0.1,1,10$, and $100 \mathrm{kHz}$. Temperature was varied continuously from 113 to $453 \mathrm{~K}$ in a Delta furnace at $2 \mathrm{~K} / \mathrm{min}$. The disc was then sawn up, with one long piece $\left(15 \times 4.4 \times 1.6 \mathrm{~mm}^{3}\right)$ used for dynamical mechanical analysis (DMA) measurements in a DMA-Q800 (TA Instruments). Data were collected in three point bending mode with a frequency range of $0.2-20 \mathrm{~Hz}$, under dynamic amplitude of up to $4 \mu \mathrm{m}$ and a static load of $0.01 \mathrm{~N}$ during continuous heating at $3 \mathrm{~K} / \mathrm{min}$. Smaller rectangular parallelepipeds were also sawn from the BZT-50BCT and BTO discs $\left(3.205 \times 3.500 \times 1.584 \mathrm{~mm}^{3}, 0.0922 \mathrm{~g}\right.$ and 3.206x3.715x1.349 $\mathrm{mm}^{3}, 0.0920 \mathrm{~g}$, respectively) for RUS.

Values of the bulk, $K$, and shear, $G$, moduli at room temperature were determined from fitting of the frequencies of 25 30 peaks in RUS spectra using Dynamic Resonance Systems (DRS) software.[22,23] These are given in Table 1, along with estimates of the porosity of each sample based on a measured density from dimensions and mass in comparison with a theoretical density based on composition and lattice parameters.[15,24]

Equipment used for the high temperature RUS measurements has been described in detail elsewhere.[23,25,26] RUS spectra of BZT-50BCT containing 50000 data points in the frequency range 0.1-1.2 MHz were collected in a high temperature furnace during heating and cooling between 291 and $540 \mathrm{~K}$ in $\sim 5 \mathrm{~K}$ steps, with a thermal equilibration time of $20 \mathrm{~min}$ at each set point. The same procedure was followed for BTO through the temperature interval 
291-500 K. In each case the sample rested lightly across its corners between the tips of two alumina buffer rods. In the low temperature instrument, each sample sat directly between the transducers across a pair of faces. Each spectrum again contained 50000 data points in the frequency range 0.1-1.2 MHz. The data reported here were collected in heating sequences from $\sim 5$ to $\sim 300 \mathrm{~K}$ in $5 \mathrm{~K}$ steps, again with a period of 20 minutes for thermal equilibration at each set point. Individual peaks in the spectra were fitted with an asymmetric Lorentzian function, within the software package Igor (WaveMetrics), to give the peak frequency, $f$, and width at half maximum height, $\Delta f$. The elastic constants scale with $f^{2}$ and the inverse mechanical quality factor, $Q^{-1}=\Delta f / f$, is a measure of acoustic loss. For a ceramic sample, the variation of $f^{2}$ effectively represents the variation of the shear modulus.

\begin{tabular}{lcccccccc}
\hline Sample & $\begin{array}{c}\text { Molar } \\
\text { mass } \\
(\mathrm{g} / \mathrm{mol})\end{array}$ & $\begin{array}{c}\text { Unit cell } \\
\text { Volume } \\
\left(\mathrm{A}^{3}\right)\end{array}$ & $\begin{array}{c}\text { Theoretical } \\
\text { density } \\
\left(\mathrm{g} / \mathrm{cm}^{3}\right)\end{array}$ & $\begin{array}{c}\text { Measured } \\
\text { density } \\
\left(\mathrm{g} / \mathrm{cm}^{3}\right)\end{array}$ & $\begin{array}{c}\text { Estimated } \\
\text { porosity } \\
(\%)\end{array}$ & $\begin{array}{c}K \\
\pm 1.3 \% \\
(\mathrm{GPa})\end{array}$ & $\begin{array}{c}\text { ( } 0.1 \% \\
(\mathrm{GPa})\end{array}$ & $\begin{array}{c}\text { Rms } \\
\text { error } \\
(\%)\end{array}$ \\
\hline $\mathrm{BZT-50BCT}$ & 222.94 & 64.28 & 5.757 & 5.189 & 9.8 & 81.2 & 30.78 & 0.41 \\
$\mathrm{BaTiO}_{3}$ & 233.2 & 64.32 & 6.019 & 5.726 & 4.9 & 95.5 & 39.58 & 0.26 \\
\hline
\end{tabular}

Table 1. Estimated density and porosity for the BTO and BZT-50BCT samples used for RUS, together with room temperature elastic moduli. The rms error is an indication of the goodness of fit between the observed and calculated frequencies of 25 - 30 resonance peaks. (2 peaks out of the first 28 were not observed from BTO and 4 out of the first 29 for BZT-50BCT).

\section{Results}

In order to confirm the sample quality, X-ray diffraction (XRD) patterns of the fabricated BZT-50BCT and pure BTO samples were collected and the peaks matched with the perovskite structure (Figure 1). No extra peaks were observed. Lattice parameters obtained from refinements using the package PowderCell were tetragonal: $a=b=3.994955 \AA$, $\mathrm{c}=4.305937 \AA$ for $\mathrm{BTO}$ and $\mathrm{a}=\mathrm{b}=4.008745 \AA, \mathrm{c}=4.021188 \AA$ for BZT-50BCT. The tetragonal shear strain $(\varepsilon=(c-a) / a)$ of BTO is around $8 \%$, i.e. much higher than the value of $0.3 \%$ obtained for BZT-50BCT. This is consistent with lower polarization anisotropy of BZT-50BCT at room temperature, in comparison pure BTO, due to proximity to the MPB. 
$[4,8,12]$

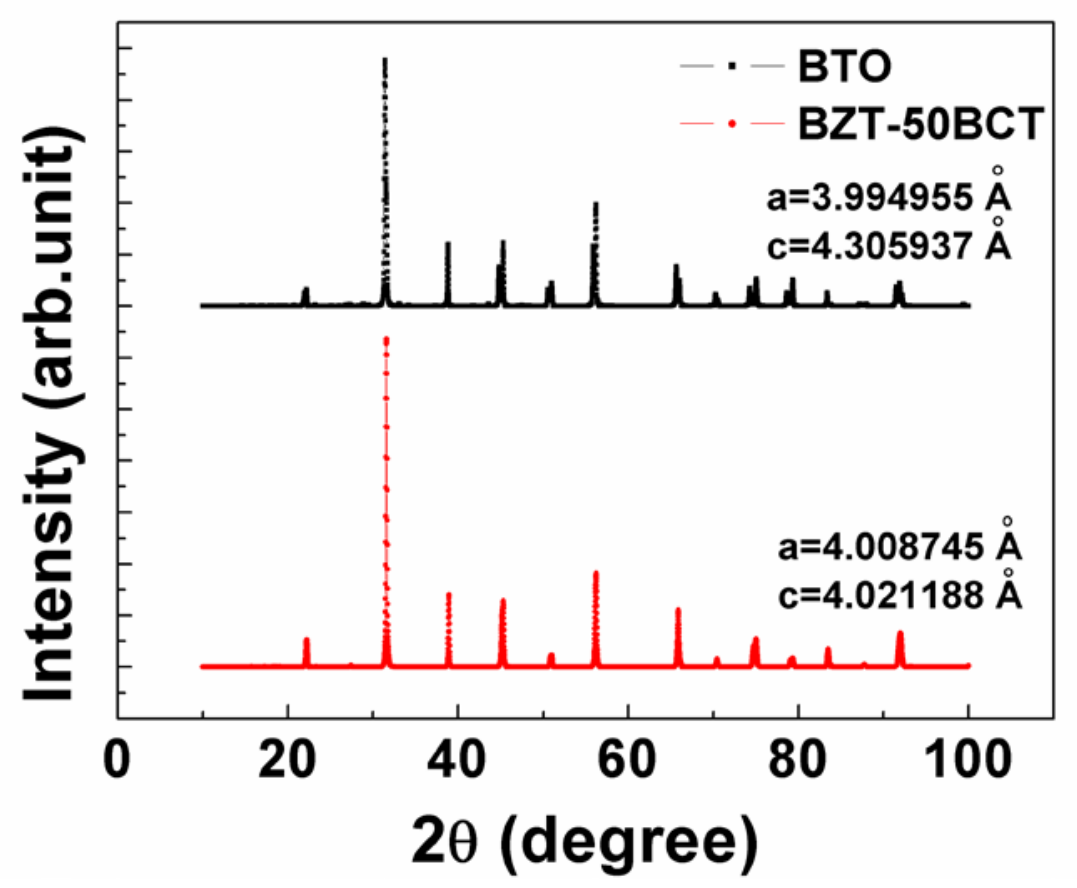

Fig.1 XRD profiles of BTO (black) and BZT-50BCT (red) measured at ambient temperature $\left(25^{\circ} \mathrm{C}\right)$, indicating that homogeneous single phase perovskites were obtained by the conventional fabrication process. Also given are the refined lattice parameters.

Scanning electronic microscopy (SEM) observations (Fig. 2) showed grain boundaries and no evidence for precipitate phases. The grain size $(30-50 \mu \mathrm{m})$ of BTO was $3 \sim 5$ times

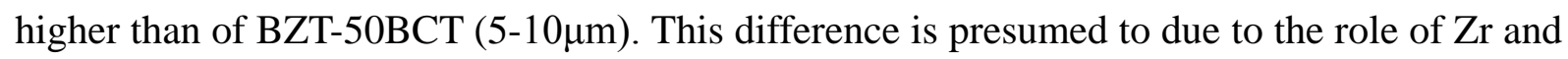
Ca dopants because the fabrication process was otherwise the same. 


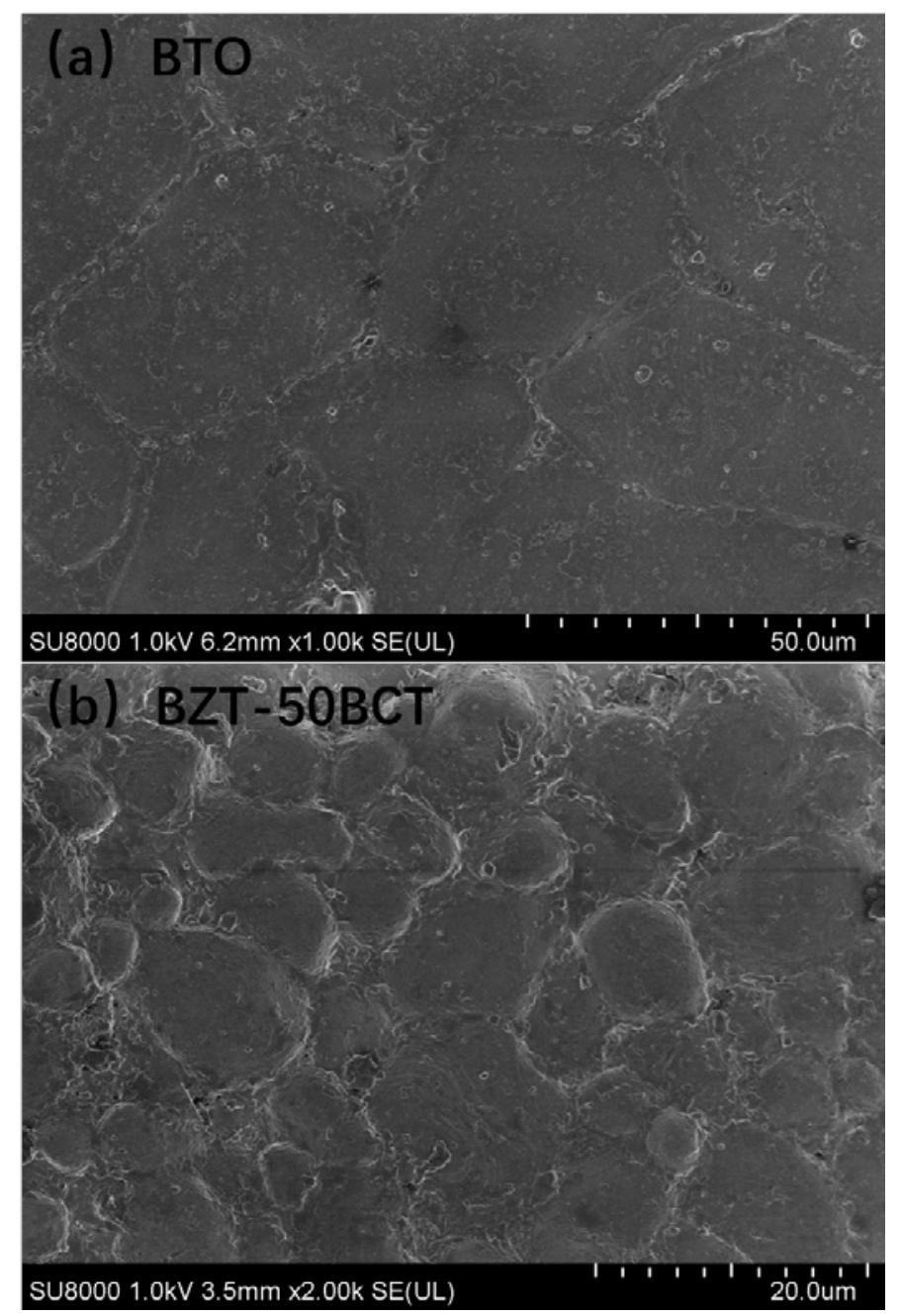

Fig.2 SEM images of (a) BTO and (b) BZT-50BCT ceramics, indicating the different grain size between BZT-50BCT and BTO samples.

Figure 3a shows a phase diagram for BZT- $x$ BCT $(x=20 \sim 60)$ determined from measurements of dielectric permittivity at multiple frequencies in the temperature range 113-453 K. There are three typical ferroelectric phases below the transition temperature, $T_{\mathrm{c}}$, from the parent cubic (C) structure. The rhombohedral (R) phase with $R 3 m$ symmetry is stable at BZT-rich compositions and the tetragonal (T) phase with $P 4 m m$ symmetry is stable at the BCT-rich end. At the intermediate composition region, i.e., $x=50$, the $\mathrm{T}$ phase can transform to the R phase spontaneously upon cooling. After some initial disagreement, an orthorhombic (O) phase is now known to occur between the $T$ and $R$ phases.[4,8,11-14,27] This can be 
smoothly connected with the stability field of the intermediate O phase of pure BTO ceramics by building the (BZT-50BCT) $\sim \mathrm{BaTiO}_{3}$ phase diagram as shown in Figure 3b. This method has been carefully discussed by Zhang et al.[12]. Dielectric permittivity (multi-frequencies) versus temperature curves of BZT-50BCT are shown in Figure 3c. Three anomalies are evident and are marked as $T_{\mathrm{c}}\left(\mathrm{C}-\mathrm{T}\right.$ transition), $T_{\mathrm{T}-\mathrm{O}}\left(\mathrm{T}-\mathrm{O}\right.$ transition), and $T_{\mathrm{O}-\mathrm{R}}(\mathrm{O}-\mathrm{R}$ transition). The highest value of the piezoelectric coefficient, $\mathrm{d}_{33} \geq 545 \mathrm{pC} / \mathrm{N}$, can be found at the T-O phase transition. $[4,8,12]$

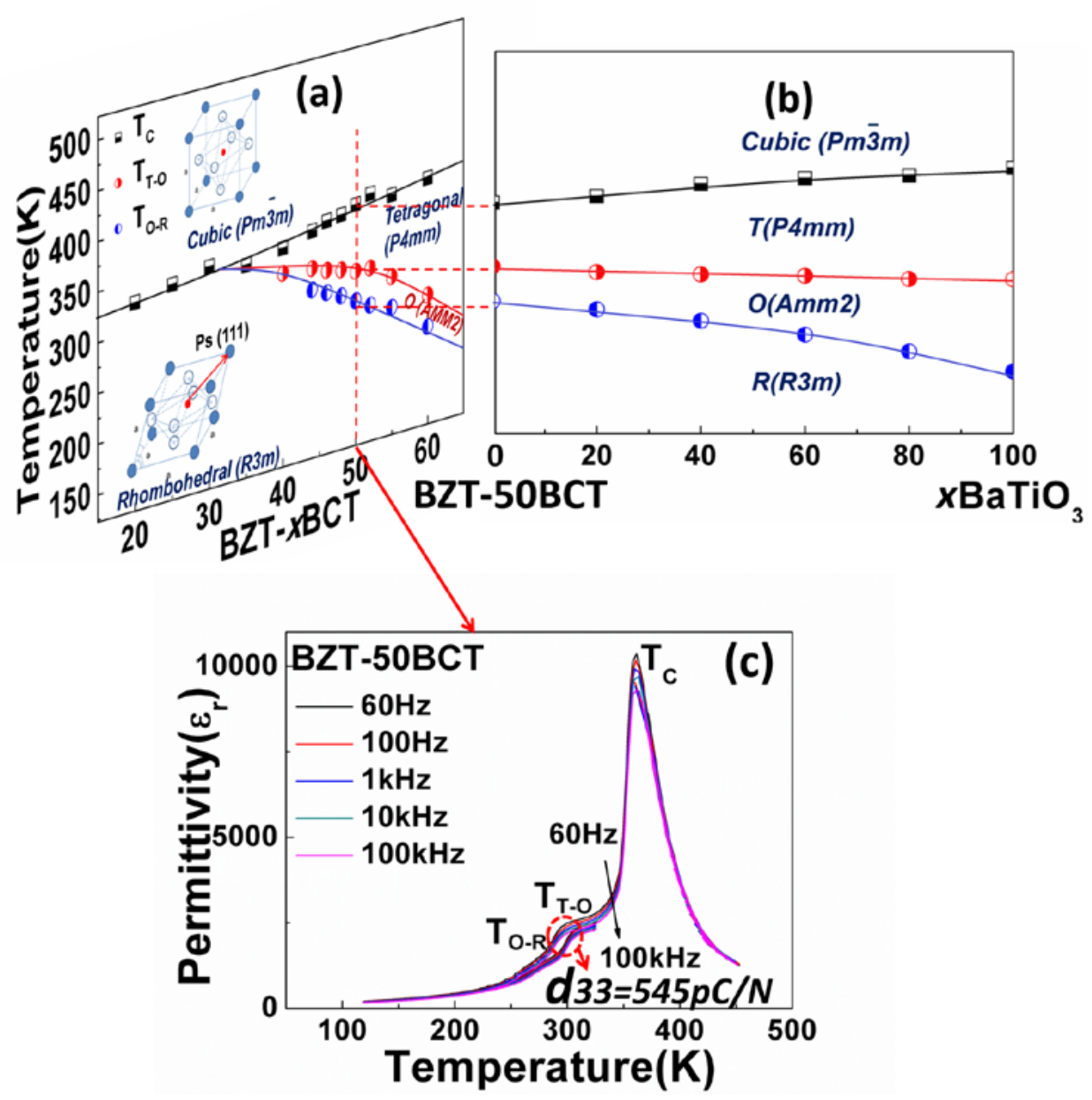

Figure 3. (a) Phase diagram of BZT- $x$ BCT system for $x=20 \sim 60$ and (b) connection from BZT-50BCT to $\mathrm{BaTiO}_{3}$, as determined from dielectric permittivity measurements. The C/T/intermediate phase/R phase regions of BZT-50BCT can be smoothly connected with the C/T/O/R phase regions of pure BTO, indicative of orthorhombic symmetry for the intermediate phase of BZT-50BCT[12]. (c) Permittivity for BZT-50BCT, measured in the frequency range $0.06 \sim 100 \mathrm{kHz}$. The highest value for the piezoelectric coefficient, $d_{33}=545 \mathrm{pC} / \mathrm{N}$ occurs at the T-O transition point $(\sim 304 \mathrm{~K})$.

Figure 4 shows RUS data from BZT-50BCT in comparison with data from BTO. Stacks of spectra, combined from the low and high temperature instruments and offset up the 
y-axis in proportion to the temperature at which they were collected, are given in Figures 4a and $4 \mathrm{~b}$, respectively. The results from fitting of the peaks indicated by arrows are given in Figures 4c and 4d. In Fig.4c, data from fitting of two resonant modes (marked by arrows in Fig.4a) are shown to give the complete pattern, especially at lower temperature (5-100 K). The results for the resonant mode at $\sim 800 \mathrm{kHz}\left(f^{2}\right.$, white-black circles, $Q^{-1}$, purple stars) have been rescaled to those for the resonance at $\sim 400 \mathrm{kHz}$. Both sets of data show a similar pattern of three minima in $f^{2}$, corresponding to the C-T, T-O and O-R transitions $(\sim 398, \sim 291$ and $\sim 202 \mathrm{~K}$ for BTO, 360, 304 and $273 \mathrm{~K}$ for BZT-50BCT), but there are subtle differences in the exact variations and the data for $Q^{-1}$ are quite different. In both cases there is substantial precursor softening of the shear modulus in the cubic phase as temperature decreases from $500 \mathrm{~K}$ towards $T_{\mathrm{c}}$. In BTO, further softening and stiffening occur around sharp minima at the transition points. These minima are rounded in the data for BZT-50BCT, particularly at the T-O and O-R transitions. Below $T_{\mathrm{O}-\mathrm{R}}$ the shear modulus of the rhombohedral structure merely stiffens smoothly with further cooling. The maximum amount of softening corresponds to 50\% at $T_{\mathrm{T}-\mathrm{O}}$ in BZT-50BCT. 

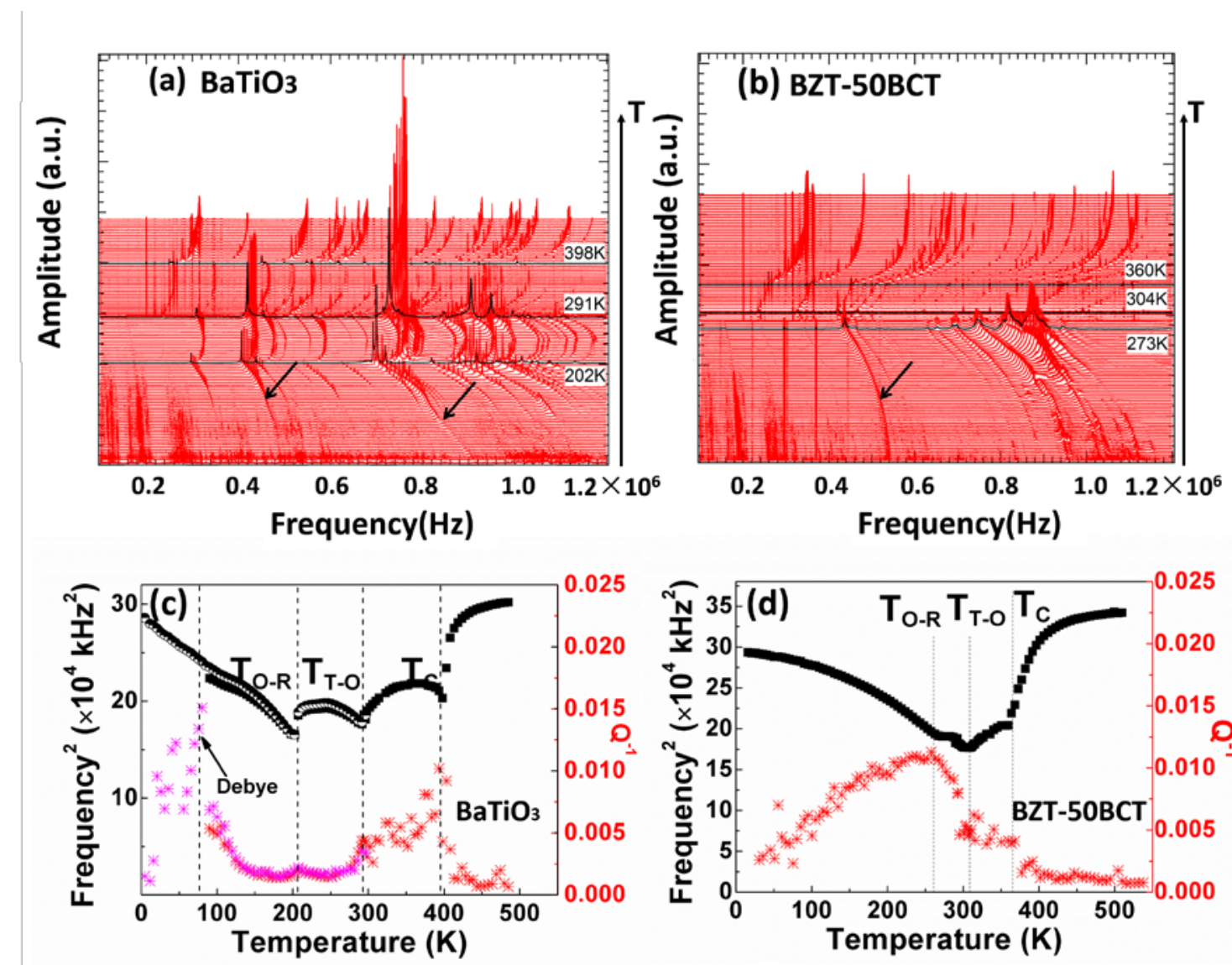

Figure 4. (a) Stack of RUS spectra for BTO ceramic collected in the range 5-500 K. (b) Stack of RUS spectra for BZT-50BCT ceramic collected in the range 5-540 K. The y-axis is the amplitude of the RUS signal, but each spectrum has been displaced in proportion to the temperature at which it was collected and the right arrows point to high temperature $(\mathrm{T})$ from ambient temperature. Weak resonance peaks above $300 \mathrm{~K}$ which are independent of temperature are from the alumina buffer rods. Similar weak peaks below $300 \mathrm{~K}$ which are also independent of temperature come from some part of the low temperature holder. (c) and (d) show the temperature evolution of the squared frequency $\left(f^{2}\right.$, black markers, left axis) and inverse mechanical quality factor $\left(Q^{-1}\right.$, red or purple markers, right axis) of BTO and BZT-50BCT, respectively. Minima in $f^{2}$ correspond to known phase transitions. A Debye loss peak appears at $\sim 70 \mathrm{~K}$ in BTO and is replaced by a wide temperature interval of high loss in BZT-50BCT.

$Q^{-1}$ in the stability field of the cubic phase is low for both samples, signifying that they are good quality ceramics without cracks or grain boundary effects which could contribute significantly to the acoustic loss. The BTO sample has an onset of increasing $Q^{-1}$ values $\sim 10-20 \mathrm{~K}$ above $T_{\mathrm{c}}$, a peak value of $\sim 0.01$ at $T_{\mathrm{c}}$ itself followed by a plateau near 0.005 in the stability field of the tetragonal structure (Fig. $4 \mathrm{c}$ ). There are small peaks at $T_{\mathrm{T}-\mathrm{O}}$ and $T_{\mathrm{O}-\mathrm{R}}$ but the loss remains low in the stability fields of the orthorhombic and rhombohedral phases, 
before what appears to be a classic Debye loss peak [28] centered on 70 K. This loss peak reveals the change of dynamic relaxation of domain walls, corresponding to effective freezing. $Q^{-1}$ also increases ahead of $T_{\mathrm{c}}$ in BZT-50BCT, but the increase at $T_{\mathrm{c}}$ is not marked by a discrete peak (Fig. 4 d). $Q^{-1}$ is approximately constant at $\sim 0.005$ between $T_{\mathrm{c}}$ and $T_{\mathrm{T}-\mathrm{O}}$ but then increases steeply through the stability field of the $\mathrm{O}$ structure reaching a rounded maximum of $\sim 0.01$ at $\sim T_{\mathrm{O}-\mathrm{R}}$. In the stability field of the $\mathrm{R}$ structure, $Q^{-1}$ gradually decreases with falling temperature and there is no sign of the Debye loss peak seen in BTO.

DMA allows dynamic elastic measurements of viscoelastic behavior at lower frequencies than RUS. The storage modulus is the real part (E') of the complex modulus $\left(E=E^{\prime}+i E^{\prime \prime}\right)$ and the imaginary part (E") reveals the elastic loss. The phase angle $(\delta)$ represents the lag between applied stress and induced strain and is given by $\tan \delta=\mathrm{E}^{\prime \prime} / \mathrm{E}$ (i.e., the internal friction). DMA data for BZT-50BCT collected in the frequency range $0.2-20 \mathrm{~Hz}$ have distinct minima in the storage modulus and maxima in $\tan \delta$ at the three transition temperatures $\left(T_{\mathrm{c}} \sim\right.$ $360 \mathrm{~K}, T_{\mathrm{T}-\mathrm{O}} \sim 304 \mathrm{~K}, T_{\mathrm{O}-\mathrm{R}} \sim 273 \mathrm{~K}$; Fig. 5). The lowest value of the storage modulus occurs at $T_{\text {T-O }}$ and amounts to a total softening of $\sim 55 \%$, with respect to the cubic phase (Fig. $5 \mathrm{a}$ ). Tan $\delta$ starts to increase $\sim 10-20 \mathrm{~K}$ above $T_{\mathrm{c}}$ and has a plateau between $T_{\mathrm{c}}$ and $T_{\mathrm{T}-\mathrm{O}}$. It then increases to a maximum at $T_{\mathrm{O}-\mathrm{R}}$ (Fig. $5 \mathrm{~b}$ ). 

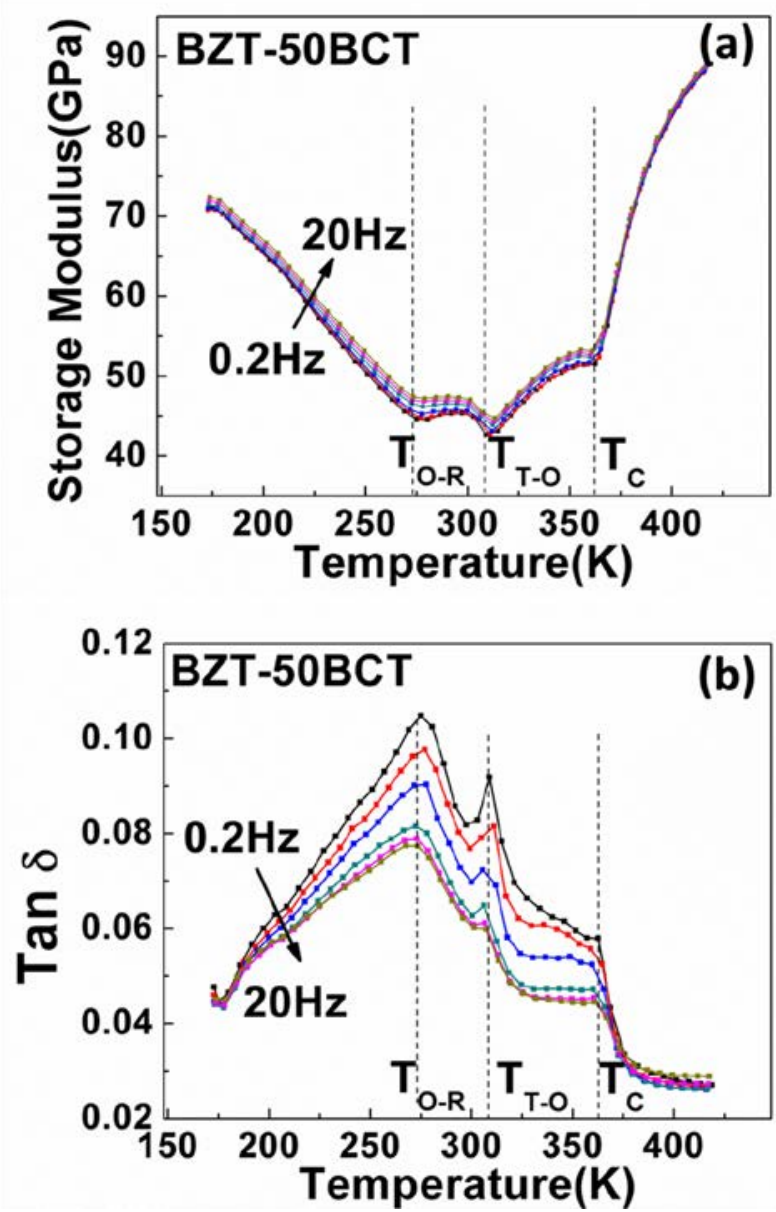

Figure 5. DMA data for BZT-50BCT in the frequency range 0.2-20 Hz: (a) Temperature dependence of the storage modulus. (b) Temperature dependence of loss, $\tan \delta$. Three minima in the storage modulus coincide with maxima or a shoulder in $\tan \delta$ at $\sim 360 \mathrm{~K}$ (C-T transition), $\sim 304 \mathrm{~K}$ (T-O transition), $\sim 273$ $\mathrm{K}$, (T-O transition).

\section{Discussion}

The overall pattern of elastic softening and stiffening observed for BZT-50BCT from both RUS and DMA measurements in the present study matches that of BTO in having three discrete phase transitions, consistent with recent determinations of the phase diagram which show the presence of a field of stability for the orthorhombic (Amm2) structure between those of the tetragonal and rhombohedral phases.[11,13,14,21] The overall pattern of softening and stiffening observed here both at $0.2-20 \mathrm{~Hz}$ and $\sim 0.1-1 \mathrm{MHz}$ is also closely comparable with what has previously been reported for BZT-50BCT from mechanical spectroscopy measurements made at $\sim 1-100 \mathrm{~Hz}[12,16,29], 8 \mathrm{kHz}[16]$ and $\sim 250 \mathrm{kHz}$.[16] The comparison with BTO is revealing, however, in that, while there are some close similarities in the mechanical properties, there are also some important differences. 
All the reported mechanical spectroscopy data for BZT-50BCT show the same precursor softening as $T \rightarrow T_{\mathrm{c}}$ from above. Classical strain coupling models predict that there will be no elastic softening ahead of the transition temperature in the case of improper ferroelastics, and the observed softening is then attributed to dynamical effects. Precursor softening evident in Figure 4 for both BTO and BZT-50BCT is the same as has been reported previously for RUS data from BTO[30-32] and is attributed largely to the effect of dynamical polar nanoregions (PNRs). These acquire a static or quasi-static component that is most likely responsible for the anelastic loss seen as increasing $\tan \delta$ and $Q^{-1}$ from $\sim 10-20 \mathrm{~K}$ above $T_{\mathrm{c}}$, and would account also for the small residual values of the piezoelectric coefficient in a $10 \mathrm{~K}$ interval above $T_{\mathrm{c}}$ reported by Acosta et al[11] and in a $30 \mathrm{~K}$ interval above $T_{\mathrm{c}}$ by Damjanovic et al.[29] This effect can also be observed in the dielectric results of BZT-50BCT in Fig.3c.

The overall elastic softening by up to $~ 50 \%$ is essentially the same in BTO and BZT-50BCT, independent of frequency (Figs. 4, 5). This is consistent with it being due to classical strain/order parameter coupling[33] and with the strength of coupling between strain and the driving order parameters being closely similar in each material. Cordero et al[16] concluded that the changes in elastic properties are intrinsic, i.e., with only small changes due to anelastic losses, and their view is supported by the present RUS data. By the same argument, it seems that rounding of the minima in elastic moduli[12,16,29] (including this study) is also intrinsic. Some of this could be due to changing of proportions of phases with different elastic properties through the two phase region of a first order transition but, if this is the case, the same would apply to BTO for which all three anomalies are sharply defined minima (Fig. 2a). If the rounding was due to variations of transition temperatures in regions of crystal with different bulk compositions, it would be expected to occur also at $T_{\mathrm{c}}$, but this too appears not to be the case. On the other hand, BZT-50BCT must inevitably contain local strain heterogeneities due to the substitutions $\mathrm{Ca}^{2+}(\mathrm{r} \sim 1.34 \AA) \leftrightarrow \mathrm{Ba}^{2+}(1.61 \AA)$ on A-sites and $\mathrm{Zr}^{4+}(0.72 \AA) \leftrightarrow \mathrm{Ti}^{4+}(0.61 \AA)$, on B-sites of the perovskite structure (radii for 12-fold and 6-fold coordination from Shannon et al.[34] For example, the diameter of strain fields around La substituted for $\mathrm{Pr}$ in $\mathrm{PrAlO}_{3}$ is $~ 15-20$ A..[35] Local strain heterogeneity and soft elastic constants might smooth out the change in strain states in going from tetragonal to orthorhombic or orthorhombic to rhombohedral but it might also enhance the propensity for 
the development of an adaptive microstructure of nanodomains, as reported, for example, by Gao et al.[8,20]Evidence for quite different dynamics between BTO and BZT-50BCT is provided by the very different patterns of acoustic loss. High loss in the stability field of the tetragonal structure of BTO is understandable in terms of the mobility under applied stress of ferroelastic twin walls. Even though $Q^{-1}$ is lower in the stability fields of the $\mathrm{O}$ and $\mathrm{R}$ phases it is still higher than for the parent cubic structure, indicating that the twin walls remain mobile (Fig. 4c). The Debye loss peak centered on $\sim 70 \mathrm{~K}$ then marks the temperature below which the twin walls become immobilized by pinning to defects. In marked contrast, the increase of $Q^{-1}$ in BZT-50BCT below $T_{\mathrm{T}-\mathrm{O}}$ to a maximum at $T_{\mathrm{O}-\mathrm{R}}$ indicates a different style of loss which is more typical of a system in which there is a wide spread of strain relaxation times. It is more similar to what is seen in $\mathrm{Pb}\left(\mathrm{Mg}_{1 / 3} \mathrm{Nb}_{2 / 3}\right) \mathrm{O}_{3}[36]$ and $\mathrm{Pb}\left(\mathrm{Fe}_{1 / 2} \mathrm{Nb}_{2 / 2}\right) \mathrm{O}_{3}[37]$ and the same explanation is likely to apply, namely that there is a range of interactions of twin walls with defects, having different local attempt frequencies and different activation energy barriers according to the local strain state.

Under the low frequency and relatively high stress conditions of DMA, the dominant mechanism of twin wall motion is likely to be forward and back motion of needle tips whereas, under the high frequency and relatively low stress conditions of an RUS experiment the dominant mechanism is likely to involve displacements of ledges along the length of the twin walls.[32,38,39] The overall form of $\tan \delta$ (Fig.3b) and $Q^{-1}$ (Fig. 4d) is broadly similar, however, suggesting that the spread of relaxation times and the pinning mechanisms are similar under both sets of conditions. On the other hand, $\tan \delta$ shows a sharp peak at $T_{\mathrm{T}-\mathrm{O}}$ while $Q^{-1}$ does not and this loss mechanism is most likely due to interfaces between coexisting tetragonal and orthorhombic regions which are mobile on a time scale of $\sim 1$ sec but not on a time scale of $\sim 10^{-6}$ secs.

For perovskite ferroelectrics, varying polarization with the displacement of cations and anions must be accompanied by changes in strain. Comparison of dielectric data with elastic compliance data allows discrimination between susceptibility with respect to changes in electric polarization and susceptibility with respect to strain. Figure 6a shows permittivity and elastic compliance $\left(1 / f^{2}\right)$ plotted on the same graph for BTO and Figure $6 \mathrm{~b}$ shows the dielectric and mechanical losses. Peaks in $1 / f^{2}, Q^{-1}$ and permittivity for the C-T transition are 
all within $\sim 1-2 \mathrm{~K}$ of $\sim 400 \mathrm{~K}$, which is consistent with the peak in $\tan \delta$. Figure 7 contains the data for BZT-50BCT plotted in an analogous way, also including the compliance (1/storage modulus) and loss ( $\tan \delta$ ) from DMA. Peaks in permittivity and all the acoustic data for the C-T transition are within $\sim 1-2 \mathrm{~K}$ of $360 \mathrm{~K}$ but the peak in dielectric $\tan \delta$ is $\sim 10 \mathrm{~K}$ lower at 352 K. The permittivity peak is also clearly wider than for the same transition in BTO implying that the transition in BZT-50BCT has more of a relaxor-like component, i.e., a larger role for PNR's. This difference is also understandable in terms of the role of significant local strain heterogeneity in BZT-50BCT which would serve to enhance and stabilize the PNR's.
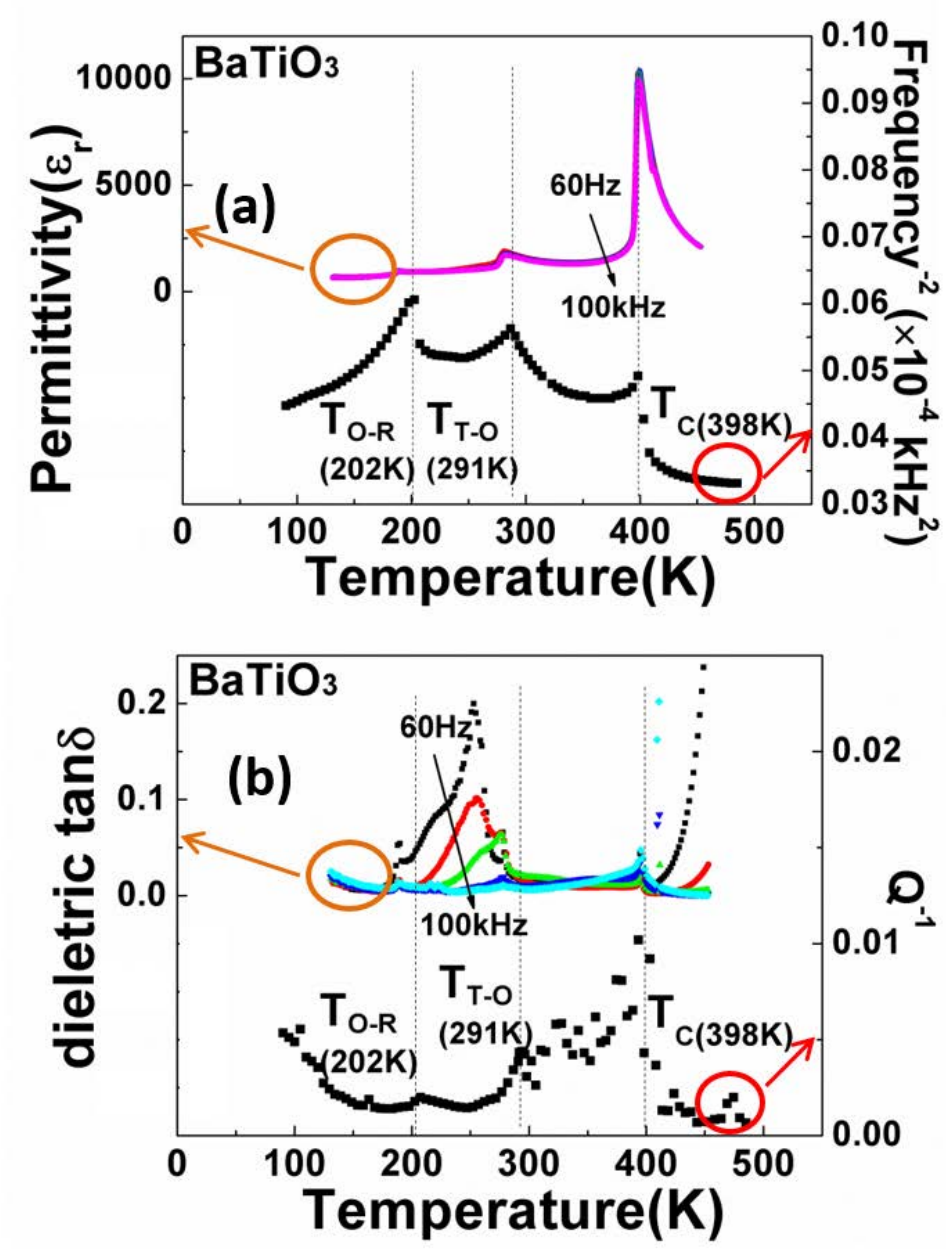

Figure 6. Comparison of results from dielectric spectroscopy and RUS for BTO: (a) permittivity ( $\varepsilon_{\mathrm{r}}$, multi colors markers, left y axis) and elastic compliance $\left(1 / f^{2}\right.$, black markers, right y axis), (b) dielectric loss $\left(\tan \delta\right.$, multi colors markers, left y axis) and acoustic loss $\left(Q^{-1}\right.$, black markers, right y axis). Dielectric data were collected during continuous cooling. There are clear and discrete anomalies associated with each of the three phase transitions. An additional dielectric loss peak occurs in the stability field of the orthorhombic structure but differs from the others in being strongly dependent on frequency. 


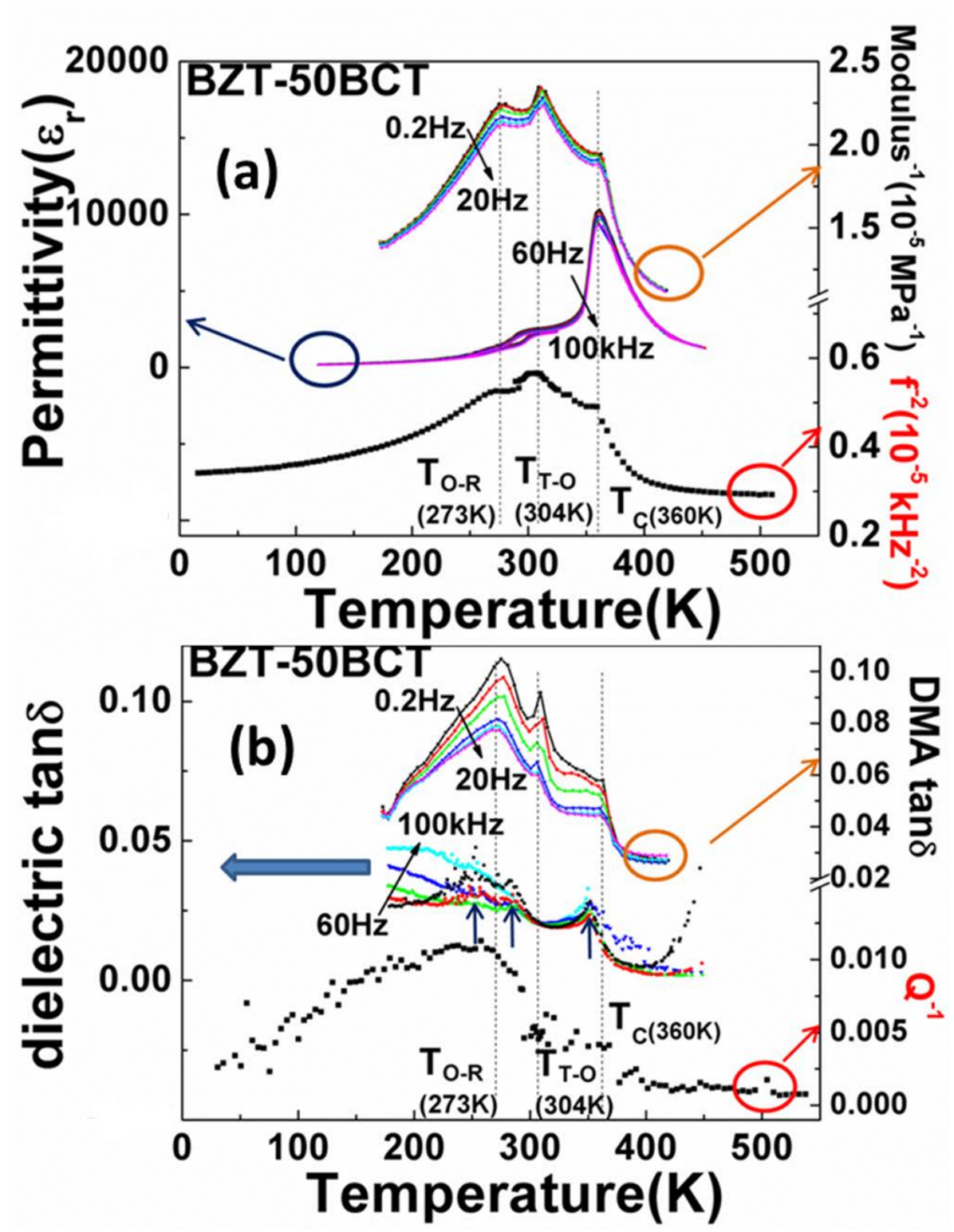

Figure 7. Comparison of dielectric spectroscopy and mechanical spectroscopy data for BZT-50BCT: (a) Dielectric permittivity (measured at $\sim 10-10^{5} \mathrm{~Hz}$ ), elastic compliance from DMA (inverse of storage modulus, measured at $\left.\sim 10^{0}-10^{1} \mathrm{~Hz}\right)$, and elastic compliance from RUS $\left(1 / f^{2}\right.$, measured at $\left.\sim 10^{5}-10^{6} \mathrm{~Hz}\right)$. (b) Dielectric loss and acoustic loss. DMA data were collected during continuous heating, dielectric data during continuous cooling. The elastic compliance data still show clear anomalies associated with the phase transitions but the permittivity has a peak only at $T_{\mathrm{c}}$. The patterns of dielectric and acoustic loss differ markedly from each other and from those seen in BTO under the same experimental conditions.

Changes in elastic constants due to intrinsic effects are understood in terms of the combined effects of order parameter susceptibility and coupling of the driving order parameter with strain.[40,41] The dielectric permittivity relates to the susceptibility with 
respect to polarization directly and this is largest at $T_{\mathrm{c}}$. Changes in strain occur at the T-O and O-R transitions and would account for the more pronounced changes in elastic properties than in the dielectric properties, therefore. (In BTO the dielectric anomalies associated with these transitions occur $\sim 10 \mathrm{~K}$ below the acoustic anomalies, but this is at least in part due to the data being collected during continuous cooling). The overall pattern of both properties at the transition points is similar and the elastic anomalies are sharply defined. In marked contrast for BZT-50BCT, anomalies in the permittivity are not in the form of discrete peaks and peaks in dielectric $\tan \delta$ (marked with arrows in Fig. 7a) are not obviously related to the transition temperatures. Furthermore, the acoustic loss is very substantially different from BTO and this must be indicative of different twin wall behaviour.

Dielectric losses are likely to be due primarily to the motion of $180^{\circ}$ walls. There is a strong frequency-dependent loss in the stability field of the orthorhombic structure of BTO which most likely arises from this mechanism. Additional dielectric loss is seen in a similar temperature interval for BZT-50BCT but the pattern is quite different. The configuration and dynamics of ferroelectric twin walls must be quite different between the two materials. Acoustic losses reflect the mobility of $90^{\circ}$ walls and, as has already been discussed, the wide interval of high loss in BZT-50BCT can be attributed to a spread of relaxation times for interactions between the ferroelastic twin walls and a matrix which is heterogeneous with respect to strain.

Figure 8 shows the further RUS data under higher temperature (360K-730K) in order to obtain and compare the Burns temperatures of BZT-50BCT and BTO case. According to previous literature[30], the Burns temperature is related to the preliminary appearing of PNRs. For elastic properties, we can define it as the first clear softening point upon cooling from high temperature. Based on this and high temperature RUS results (see figure 8), the clear Burns temperature $\left(\mathrm{T}_{\mathrm{B}}\right)$ in BZT-50BCT is around $544 \mathrm{~K}$. As comparison, it is around $510 \mathrm{~K}$ for BTO case. The clearly observed $\mathrm{T}_{\mathrm{B}}$ of BZT-50BCT is higher than the BTO case. This is of great possibility related to the discovered strain heterogeneity state of BZT-50BCT, which may benefit the formation of PNRs. 


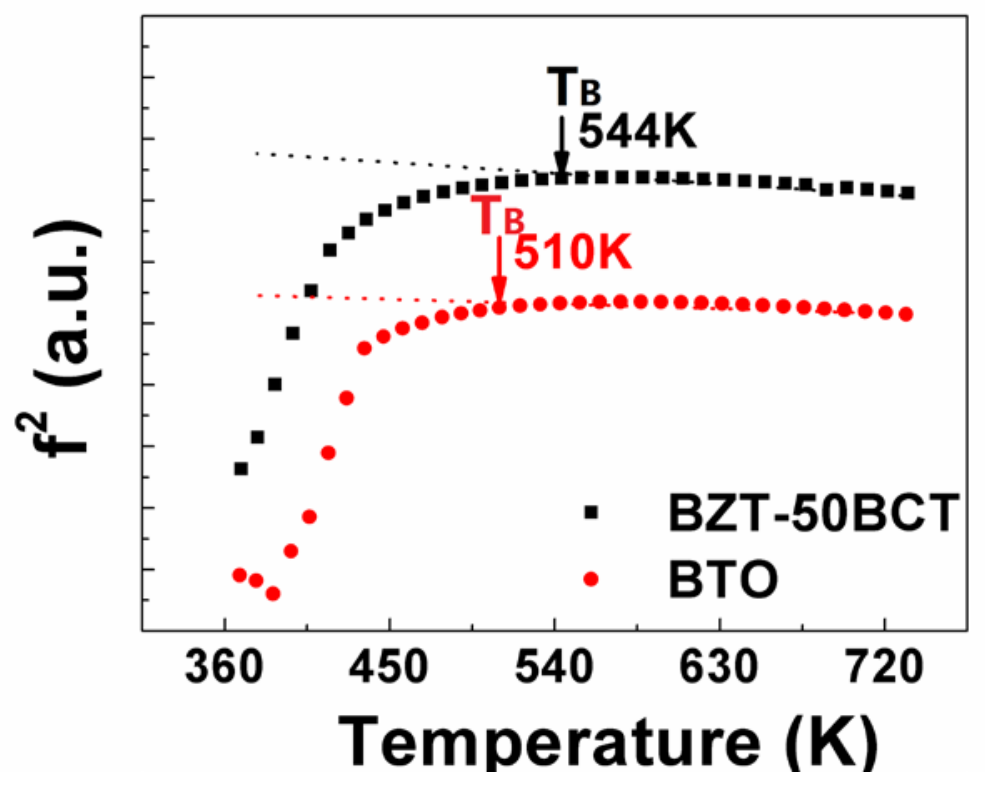

Fig.8 The temperature evolution of $\mathrm{f}^{2}$ (proportional to shear elastic modulus) in the temperature range of $360 \mathrm{~K}-730 \mathrm{~K}$ for the BZT-50BCT and BTO polycrystalline ceramics. The Burns temperature $\left(T_{B}\right)$ is defined as the preliminary softening of PNRs. The $T_{B}$ of BZT-50BCT (544K) is clearly higher than that of BTO case (510K). This may be due to the strain heterogeneity state of BZT-50BCT

In terms of the properties which particularly lead to high piezoelectric coefficients, Acosta et al showed that the highest value of $d_{33}$ occurs at $T_{\mathrm{T}-\mathrm{O}}$ in BZT-50BCT and that this coincides with the maximum value of the elastic compliance, $s_{33}$.[11] They showed also that the elastic compliance is more important than elastic anisotropy in determining the piezoelectric response. Here we have extended the frequency range of elastic measurements to confirm the results of Cordero et al[16] for the minimum shear modulus (maximum compliance) as well as the wider pattern of stiffening/softening with temperature. The lack of significant frequency dependence even across this wider range also supports the view of Cordero et al[16] that the changes are intrinsic in origin. The rounded minimum at $T_{\mathrm{T}-\mathrm{O}}$ in BZT-50BCT is also independent of frequency and therefore intrinsic, signifying an important difference from BTO. The much more obvious differences in dielectric and acoustic loss between BTO and BZT-50BCT testify to a spectrum of relaxation times for the ways in which ferroelastic $\left(90^{\circ}\right)$ twin walls interact with defects whether the motion is of needle tips of more 
local displacements.

\section{Conclusion}

The ferroelectric transitions and twin wall dynamics in the lead-free ceramic $\mathrm{Ba}\left(\mathrm{Zr}_{0.2} \mathrm{Ti}_{0.8}\right) \mathrm{O}_{3^{-}}$ 50( $\left(\mathrm{Ba}_{0.7} \mathrm{Ca}_{0.3}\right) \mathrm{TiO}_{3}\left(\mathrm{BZT}-50 \mathrm{BCT}\right.$ with piezoelectric coefficient $\left.\mathrm{d}_{33}>545 \mathrm{pC} / \mathrm{N}\right)$ have been investigated by dielectric and mechanical spectroscopies under different frequencies. Results from dielectric spectroscopy in the frequency range $60 \mathrm{~Hz}-100 \mathrm{kHz}$, dynamical mechanical analysis in the frequency range $0.2-20 \mathrm{~Hz}$ and resonant ultrasound spectroscopy in the frequency range $\sim 0.1-1.2 \mathrm{MHz}$ confirm the existence of three phase transitions with falling temperature, at $\sim 360 \mathrm{~K}$ (cubic-tetragonal), $\sim 304 \mathrm{~K}$ (tetragonal-orthorhombic) and $\sim 273 \mathrm{~K}$ (orthorhombic-rhombohedral). Based on the comparison of permittivity and elastic behavior of BZT-50BCT and BTO under wide frequency range, it is concluded that these important differences between BZT-50BCT and BTO case are most likely due to unit cell scale strain heterogeneity associated with replacing cations on the A and B sites with others having different radii. Such local heterogeneity on a unit cell enhances the more relaxor-like characteristics of BZT-50BCT (higher Burns temperature) and is likely also to play a role in favouring the development of an adaptive microstructure of nanotwin domains at the T-O boundary and resulting in the enhanced piezoelectric properties.

\section{Acknowledgements}

The authors gratefully acknowledge the funding support of the China Scholarship Council, the National Basic Research Program of China (Grant No. 2012CB619401), National Natural Science Foundation of China (Grant Nos. 51431007, 51372195, 51321003, 51231008 and 51320105014), as well as the CSS project (Grant No. YK2015-0602006). RUS facilities in Cambridge have been funded by the Natural Environment Research Council and the Engineering and Physical Sciences Research Council of Great Britain (grant nos. (NE/B505738/1, NE/F017081/1, EP/I036079/1). The authors are grateful to Mr. Yonggang Yao for kind contributions to the DMA experiments and discussion. 


\section{References}

[1] Y. Saito, H. Takao, T. Tani, T. Nonoyama, K. Takatori, T. Homma, T. Nagaya, and M. Nakamura, Nature 432, 84 (2004).

[2] T. Takenaka and H. Nagata, J. European Ceram. Soc. 25, 2693 (2005).

[3] T. R. Shrout and S. J. Zhang, Journal of Electroceramics 19, 113 (2007).

[4] W. Liu and X. Ren, Physical Review Letters 103, 257602 (2009).

[5] G. A. Rossetti, A. G. Khachaturyan, G. Akcay, and Y. Ni, Journal of Applied Physics 103, 114113 (2008).

[6] M. Ahart et al., Nature 451, 545 (2008).

[7] D. Damjanovic, Applied Physics Letters 97, 062906 (2010).

[8] J. Gao et al., Applied Physics Letters 99, 092901 (2011).

[9] M. Porta and T. Lookman, Phys. Rev. B 83, 174108 (2011).

[10] H. Bao, C. Zhou, D. Xue, J. Gao, and X. Ren, Journal of Physics D: Applied Physics 43, 465401 (2010).

[11] M. Acosta, N. Khakpash, T. Someya, N. Novak, W. Jo, H. Nagata, G. A. Rossetti, and J. Rödel, Phys. Rev. B 91, 104108 (2015)

[12] L. Zhang et al., Applied Physics Letters 105, 162908 (2014).

[13] M. Acosta, N. Novak, W. Jo, and J. Rödel, Acta Materialia 80, 48 (2014).

[14] D. S. Keeble, F. Benabdallah, P. A. Thomas, M. Maglione, and J. Kreisel, Applied Physics Letters 102, 092903 (2013).

[15] A. B. Haugen, J. S. Forrester, D. Damjanovic, B. Li, K. J. Bowman, and J. L. Jones, Journal of Applied Physics 113, 014103 (2013)

[16] F. Cordero, F. Craciun, M. Dinescu, N. Scarisoreanu, C. Galassi, W. Schranz, and V. Soprunyuk, Applied Physics Letters 105, 232904 (2014). 
[17] M. C. Ehmke, S. N. Ehrlich, J. E. Blendell, and K. J. Bowman, Journal of Applied Physics 111, 124110 (2012).

[18] D. R. J. Brandt, M. Acosta, J. Koruza, and K. G. Webber, Journal of Applied Physics 115, 204107 (2014).

[19] H. Guo, B. K. Voas, S. Zhang, C. Zhou, X. Ren, S. P. Beckman, and X. Tan, Phys. Rev. B 90, 014103 (2014).

[20] J. Gao, L. Zhang, D. Xue, T. Kimoto, M. Song, L. Zhong, and X. Ren, Journal of Applied Physics 115, 054108 (2014).

[21] J. Gao, X. Hu, L. Zhang, F. Li, L. Zhang, Y. Wang, Y. Hao, L. Zhong, and X. Ren, Applied Physics Letters 104, 252909 (2014).

[22] A. Migliori and J. L. Sarrao, Resonant ultrasound spectroscopy: applications to physics, materials measurements, and nondestructive evaluation (Wiley, 1997).

[23] R. E. A. McKnight, M. A. Carpenter, T. W. Darling, A. Buckley, and P. A. Taylor, American Mineralogist 92, 1665 (2007).

[24] G. H. Kwei, A. C. Lawson, S. J. L. Billinge, and S. W. Cheong, The Journal of Physical Chemistry 97, 2368 (1993).

[25] A. Migliori, J. L. Sarrao, W. M. Visscher, T. M. Bell, M. Lei, Z. Fisk, and R. G. Leisure, Physica B: Condensed Matter 183, 1 (1993).

[26] E. A. M. Ruth, T. Moxon, A. Buckley, P. A. Taylor, T. W. Darling, and M. A. Carpenter, Journal of Physics: Condensed Matter 20, 075229 (2008).

[27] D. Xue, Y. Zhou, H. Bao, C. Zhou, J. Gao, and X. Ren, Journal of Applied Physics 109, 054110 (2011).

[28] A. S. Nowick, Anelastic Relaxation in Crystalline Solids (Academic Press, 1972).

[29] D. Damjanovic, A. Biancoli, L. Batooli, A. Vahabzadeh, and J. Trodahl, Applied Physics Letters 100, 192907 (2012)

[30] E. K. H. Salje, M. A. Carpenter, G. F. Nataf, G. Picht, K. Webber, J. Weerasinghe, S. Lisenkov, and L. Bellaiche, 
Phys. Rev. B 87, 014106 (2013).

[31] O. Aktas, M. A. Carpenter, and E. K. H. Salje, Applied Physics Letters 103, 142902 (2013).

[32] M. A. Carpenter, Journal of Physics: Condensed Matter 27, 263201 (2015).

[33] M. A. Carpenter and E. K. Salje, European Journal of Mineralogy 10, 693 (1998).

[34] R. t. Shannon, Acta Crystallographica Section A: Crystal Physics, Diffraction, Theoretical and General Crystallography 32, 751 (1976).

[35] M. A. Carpenter, R. E. A. McKnight, C. J. Howard, Q. Zhou, B. J. Kennedy, and K. S. Knight, Phys. Rev. B 80, 214101 (2009).

[36] M. A. Carpenter, J. F. J. Bryson, G. Catalan, S. J. Zhang, and N. J. Donnelly, Journal of Physics: Condensed Matter 24, 045902 (2012).

[37] M. A. Carpenter et al., Journal of Physics: Condensed Matter 27, 285901 (2015).

[38] M. A. Carpenter and Z. Zhang, Geophysical Journal International 186, 279 (2011).

[39] E. K. H. Salje, X. Ding, Z. Zhao, T. Lookman, and A. Saxena, Phys. Rev. B 83, 104109 (2011).

[40] J. C. Slonczewski and H. Thomas, Phys. Rev. B 1, 3599 (1970).

[41] W. Rehwald, Advances in Physics 22, 721 (1973). 